\title{
Meta-analysis of postoperative adjuvant chemotherapy without radiotherapy in early stage non-small cell lung cancer
}

This article was published in the following Dove Press journal:

OncoTargets and Therapy

4 August 2015

Number of times this article has been viewed

\author{
Yuan-Yuan Chen* \\ Lin-Wei Wang* \\ Shu-YiWang \\ Bi-Bo Wu \\ Zhen-Meng Wang \\ Fang-Fang Chen \\ Bin Xiong
}

Department of Oncology, Zhongnan Hospital of Wuhan University, Hubei Key Laboratory of Tumor Biological Behaviors and Hubei Cancer Clinical Study Center, Wuhan, Hubei, People's Republic of China

*These authors contributed equally to this work
Correspondence: Bin Xiong Department of Oncology, Zhongnan Hospital of Wuhan University, Hubei Key Laboratory of Tumor Biological Behaviors and Hubei Cancer Clinical Study Center, No 169 Donghu Road, Wuchang District, Wuhan 43007I, Hubei Province, People's Republic of China

Tel $+86027678|3| 52$

Fax +86 027 678I2829

Email binxiong1961@whu.edu.cn
Background: Many clinical trials have confirmed that postoperative adjuvant therapy can prolong survival of non-small cell lung cancer. However, the efficiency of postoperative chemotherapy without radiotherapy is unclear, especially in early stage (stages I and II). We aimed to assess the effect of postoperative chemotherapy without radiotherapy in early stage patients.

Methods: Databases and manual searches were adopted to identify eligible randomized control trials. Hazard ratio (HR) was used to assess the advantage of disease-free survival (DFS) and overall survival (OS) by fixed or random-effects models.

Results: Fourteen trials with 3,923 patients were included based on inclusion criteria. Compared with surgery alone, postoperative chemotherapy significantly improved DFS and OS with HR of $0.71(P=0.005)$ and $0.74(P<0.00001)$, respectively. Subgroup analysis showed both cisplatin-based (HR: $0.75, P<0.0001$ ) and single tegafur-uracil (UFT) chemotherapy (HR: 0.72, $P=0.002)$ yielded significant survival benefits, but the latter did not improve DFS (HR: 1.04, $P=0.81)$. Indirect treatment comparison showed cisplatin-based chemotherapy was superior to single UFT in DFS, but comparable in OS. The benefits of postoperative chemotherapy were maintained in patients in stage I (HR: $0.74, P<0.00001)$ and IB (HR: $0.74, P=0.0003$ ), but not in stage IA, although the trend supported chemotherapy (HR: $0.76, P=0.43$ ).

Conclusion: This meta-analysis demonstrates that postoperative chemotherapy without radiotherapy improves survival of stage I-II, I, and IB non-small cell lung cancer patients, but not for IA. Meanwhile, efficacy of cisplatin-based chemotherapy is comparable to single UFT in OS, but better in DFS, which should be paid more attention in future clinical practice.

Keywords: postoperative chemotherapy, disease-free survival, overall survival

\section{Introduction}

Lung cancer is the most common malignant tumor and the leading cause of cancer-related death in the world. ${ }^{1}$ More than 1.5 million new cases of lung cancer are diagnosed every year, approximately $80 \%$ of which are non-small cell lung cancer (NSCLC). ${ }^{2}$ The morbidity is rapidly increasing mainly due to environmental pollution and unhealthy lifestyles (eg, smoking, occupational exposure, diet). ${ }^{3}$ Although surgery is the recommended treatment for NSCLC, only one-third of patients are suitable for surgery when they are diagnosed. ${ }^{4}$ Unfortunately, 5-year overall survival (OS) rate of all stages is approximately $11 \%-15 \%{ }^{5,6}$ Even among early stage (I/II) NSCLC patients who only undergo surgical resection, the 5-year OS rate is just $45.1 \% .^{7}$ These rates indicate that postoperative treatment strategies (eg, chemotherapy, radiotherapy) are essential to improve NSCLC patients' prognosis. 
Chemotherapy plays a crucial role in comprehensive NSCLC therapy. Currently, cisplatin-based drugs are the most widely used chemotherapy medicine for NSCLC. Additionally, tegafur-uracil (UFT) is another oral chemotherapy agent popular in Japan because it is associated with mild toxicity. It has been demonstrated that chemotherapy regimens based on these two drug types can significantly improve the prognosis of advanced stage (III/IV) NSCLC patients. ${ }^{8-10}$ However, whether they are beneficial for early stage (I/II) NSCLC patients still remains controversial, especially for stage I patients. ${ }^{10-15}$ For instance, the updated NCIC-JBR $10^{15}$ data with a median follow-up of 9.3 years demonstrated that cisplatin-based chemotherapy improved the survival of stage IB-II patients by $11 \%$, and subgroup analysis showed that the survival advantage was maintained in stage II patients, but not in stage IB disease. But trials conducted by Roselli et al ${ }^{14}$ indicated that postoperative cisplatin-based chemotherapy significantly improved survival of stage IB patients. Similarly, randomized controlled trials (RCTs) on postoperative chemotherapy with UFT performed by the North-east Japan Study Group for Lung Cancer Surgery (NJSG) ${ }^{11}$ and Japan Lung Cancer Research Group (JLCRG) ${ }^{12}$ also reached opposing conclusions.

Radiotherapy is also a widely used technology in cancer treatment. A previous meta-analysis ${ }^{16}$ showed that postoperative radiotherapy had a detrimental effect on survival. However, most of the current studies combined chemotherapy and radiotherapy simultaneously, such as the famous ALPI ${ }^{17}$ and IALT ${ }^{18}$ studies. The only study on postoperative chemotherapy alone was conducted by NSCLC Meta-analyses Collaborative Group and utilized many outdated regimens. ${ }^{19}$ Therefore, the effect of modern postoperative chemotherapy alone in NSCLC is unclear. Herein, we performed a new meta-analysis to investigate the survival benefits conferred by adjuvant chemotherapy without radiotherapy in early stage NSCLC patients.

\section{Patients and methods}

\section{Search strategy and eligibility criteria}

Eligible RCTs that compared surgery plus postoperative chemotherapy versus surgery alone and published in English language were identified by searching the PubMed (http:// www.ncbi.nlm.nih.gov/pubmed), Embase (http://www. embase.com/info/helpfiles/), and Science Direct (http:// www.elsevier.com/online-tools/sciencedirect) databases. We also manually searched the reference lists of relevant meta-analyses and reviews. The keywords included: "nonsmall cell lung cancer", "surgery", "resection", "lobectomy", "pleurectomy", “chemotherapy", "adjuvant", "therapy", "early", "I", "II", "1", "2", and "random*". Eligible patients had to meet the following criteria: 1) histologically confirmed NSCLC (including adenocarcinoma, squamous cell carcinoma, and large cell carcinoma) with radically resection; 2) pathologic stage I and/or II with an Eastern Cooperative Oncology Group performance status of 2 or less; 3 ) no major organ (liver, kidney, or heart) dysfunction; 4) no preoperative anticancer treatment; 5) no other cancer site besides lung; and 6) randomly assigned to surgery followed by adjuvant chemotherapy (chemotherapy group) and surgery alone (control group). Trials that evaluated other adjuvant therapies (radiotherapy, endocrine therapy, and immunotherapy) and neoadjuvant therapy were excluded from this meta-analysis. To update the data, RCTs published in a previous meta-analysis ${ }^{20}$ in 1995 were excluded from this study; only RCTs published after January 1, 1992 were enrolled.

\section{Data extraction}

Two reviewers (Yuan-Yuan Chen and Lin-Wei Wang) independently extracted the following data from each enrolled trial: publication year, first author, pathologic stage, median follow-up time, treatment compliance, number of patients, chemotherapy regimen, and toxicity data. If provided in the trial, the $P$-value and hazard ratio (HR) at 5 years obtained from Cox regression model were used directly in this metaanalysis. If not available, approximations of HR estimates were indirectly calculated based on the correlative statistics (number of observed and total events, $P$-value, 95\% confidence interval $[\mathrm{CI}]$ ) using the methods described by Tierney et al. ${ }^{21}$ Alternatively, data was extracted from published Kaplan-Meier curves. To further analyze the effects of prognostic factors on survival, subgroup analyses were carried out according to pathologic stage and adjuvant therapy type. Any discrepancy was resolved by discussing with another author (Bi-Bo Wu).

\section{Quality assessment}

The quality of the included RCTs was evaluated using the Cochrane Collaboration's risk of bias tool, ${ }^{22}$ which included adequate sequence generation, allocation concealment, blinding of participants and personnel, blinding of outcome assessment, incomplete outcome data, selective outcome reporting, and other bias. Each item was classified as low, high, and unclear risk of bias, and then a summary assessment of each included trial was graded as A, B, or C. Two reviewers concurrently checked the risk of bias. 


\section{Statistical analysis}

Review Manager (Version 5.2; The Cochrane Collaboration, Copenhagen, Denmark) was used for statistical analysis. Selection of fixed-effects or random-effects model was determined by statistical heterogeneity among the included trials, which was evaluated using the chi-square test and quantified using $I^{2}$ statistic. The existence of homogeneity was considered unreasonable for $P>50 \%$ and $P<0.10$. In general, HR $>1$ implied that the chemotherapy group had worse prognosis than the control group. Comparisons between cisplatin-based and single UFT chemotherapy used indirect treatment comparison (ITC) software (Version 1.0; Canadian Agency for Drugs and Technologies in Health, Ottawa, Canada) proposed by Bucher et $\mathrm{a}^{23}$ which estimated the relative effects of treatment $A$ and treatment $C$ through the common control treatment B. Finally, publication bias was evaluated by funnel plots and further quantified by Begg's and Egger's tests ${ }^{24,25}$ with STATA software (Version 12.0; StataCorp LP, College Station, TX, USA). Sensitivity analysis was also conducted using STATA. Two-sided $P$-value $<0.05$ was considered statistically significant.

\section{Results}

\section{Characteristics of included RCTs}

There were 580 trials searched from databases and six from relative references lists. We identified 25 potentially eligible trials, eleven of which were excluded from our meta-analysis, including four trials that lacked survival data regarding early stage NSCLC, five trials where patients received postoperative chemotherapy plus radiotherapy, and two trials that were published previously ${ }^{26,27}$ but had been updated afterward. ${ }^{15,28}$ Finally, 14 RCTs with 3,923 patients (chemotherapy group: 1,987 patients; control group: 1,936 patients) were included in this analysis. ${ }^{11-15,28-36}$ Two chemotherapy groups were separately compared with the same control group in a study by Imaizumi et $\mathrm{al}^{32}$; so we treated this trial as two independent studies. To avoid repeat counting, the control group in these two studies was counted once in the analytical process. The screening process was briefly shown in Figure 1. The available median follow-up time and chemotherapy compliance were ranged from 51 to 120 months and $53 \%$ to $86 \%$, respectively. The baseline characteristics of chemotherapy group and control group are balanced and summarized in Table 1.

All RCTs included a statement about randomization, and detailed descriptions were listed in eleven trials. Blinding of participants and personnel assessment was only described in one trial. ${ }^{14}$ However, it was not always feasible to blind in studies involving surgery. No quality difference was observed in the included RCTs except for in the study of Strauss et $\mathrm{al}^{35}$ which had a higher risk than others due to early termination (data not shown).

\section{Overall analysis of survival}

Data for 5-year disease-free survival (DFS) was available in eleven trials ${ }^{11,12,14,28,29,31-33,35-37}$ with a total of 2,937 patients. Although six trials ${ }^{11,12,28,35-37}$ showed that DFS was not significantly increased in chemotherapy group, the combined

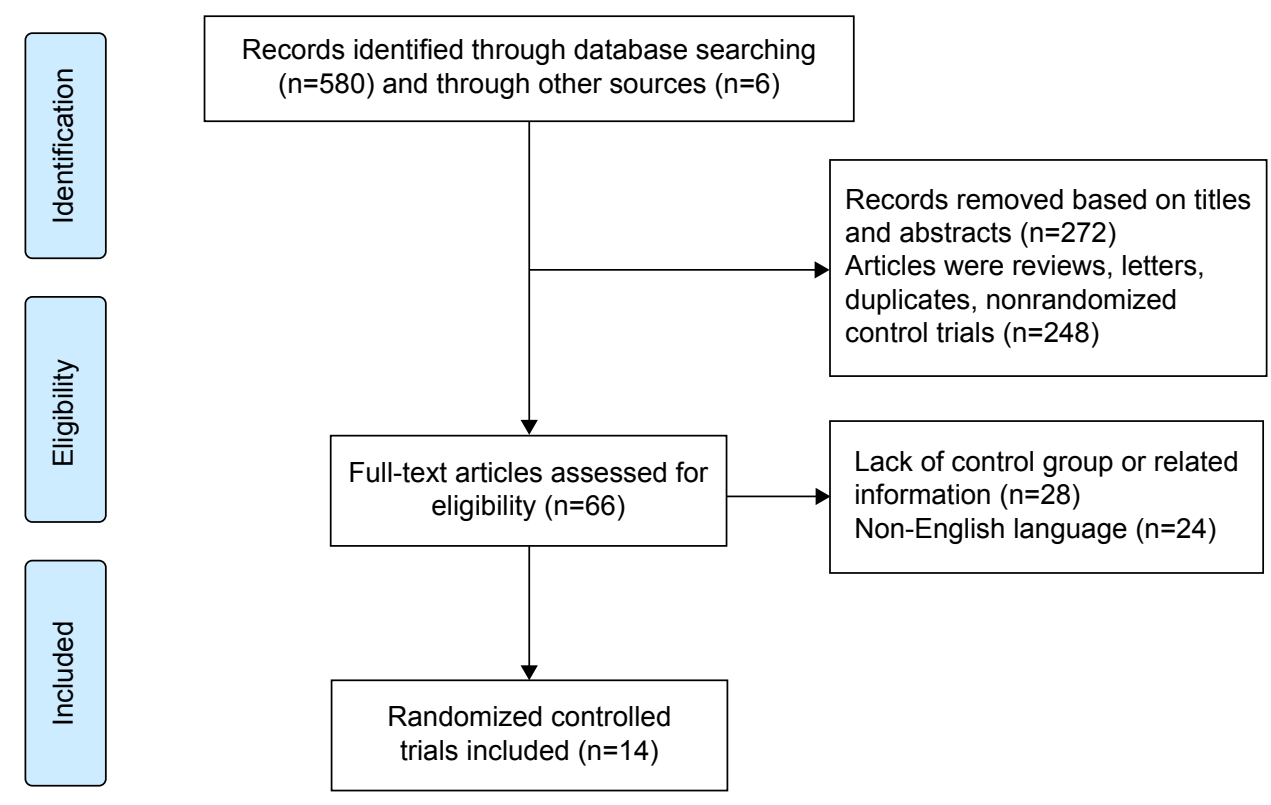

Figure I Flow chart of screening process. 


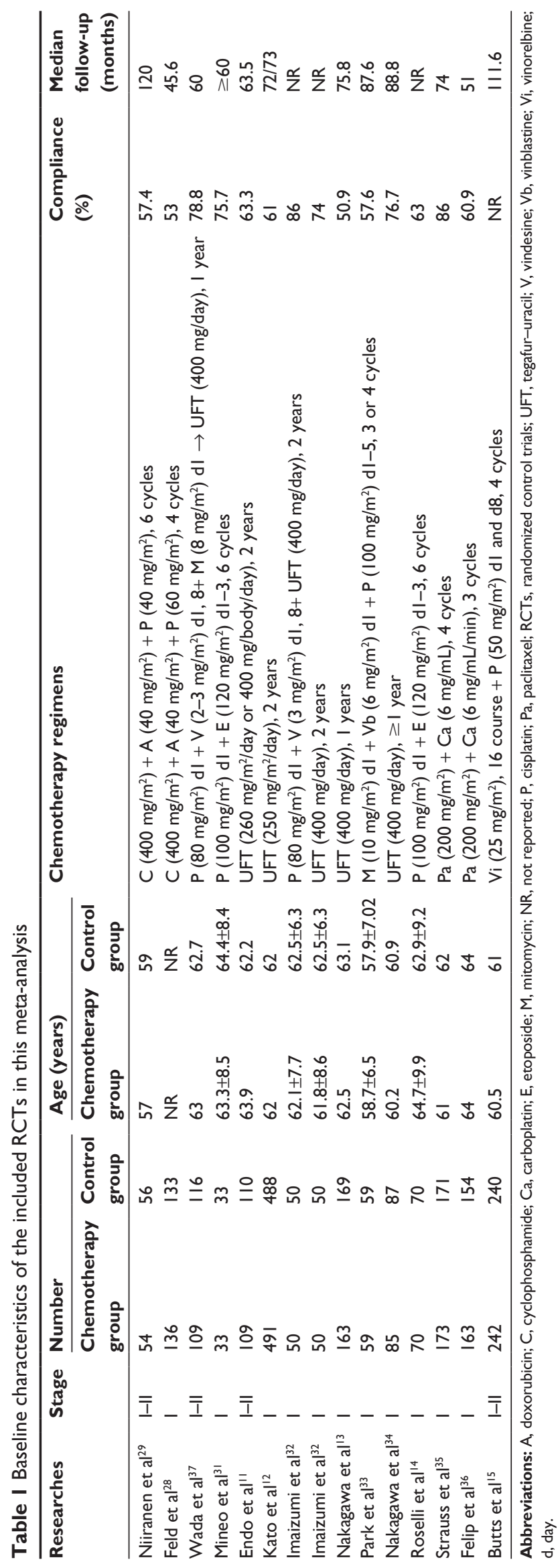

analysis displayed that survival benefit was achieved with postoperative chemotherapy $(P=0.005$; Figure $2 \mathrm{~A})$, and the recurrence risk was greater in the control group compared to the chemotherapy group ( $41.3 \%$ versus $32.2 \%$ ). Because significant heterogeneity existed among trials $\left(I^{2}=71 \%\right)$, random-effects model was adopted and achieved a combined HR of 0.71 (95\% CI, 0.57-0.90). There was evidence of publication bias by Egger's test $(P=0.029)$.

There were 13 trials ${ }^{11-15,28-35}$ (3,656 patients) with complete OS information. The analysis showed that the survival of chemotherapy group was significantly better than control group without heterogeneity in trials $\left(P<0.00001, I^{2}=0 \%\right)$. The combined HR with fixed-effects model was 0.74 (95\% CI, 0.67-0.83; Figure 2B), which represented a 26\% relative reduction of death risk in chemotherapy group. No publication bias was detected by Begg's and Egger's tests.

\section{Subgroup analysis of survival by stage}

Eight trials ${ }^{12,14,28,31-33,35,36}$ (2,833 patients) allowed for a quantitative aggregation analysis of DFS for stage I, among which three trials $\mathrm{s}^{14,31,35}$ (550 patients) reported available information on stage IB. The combined HR of stages I and IB was 0.68 $(P=0.002$; Figure $3 \mathrm{~A})$ and $0.55(P=0.02$; Figure $3 \mathrm{~B})$, respectively. An evaluation of DFS for stage IA and II patients could not be conducted due to the limited data. In terms of OS, survival in the chemotherapy group was significantly improved for patients with stage I (HR: 0.74, $P<0.00001$; Figure 4A) and IB (HR: 0.74, $P=0.0003$; Figure 4B). There was no statistically significant benefit for stage IA patients (HR: $0.76, P=0.43$; Figure $4 \mathrm{C}$ ), though there was a positive trend for improved survival in the chemotherapy group. No publication bias was detected by Begg's and Egger's tests. In this analysis, the OS data on stage II patients was not sufficient to perform a reliable analysis.

\section{Subgroup analysis of survival by chemotherapy regimens}

Applicable DFS information was analyzed in nine cisplatinbased trials (1,689 patients) and three single UFT trials (1,298 patients). Cisplatin-based chemotherapy showed results consistent with the overall DFS, which favored postoperative chemotherapy (HR: $0.61,95 \% \mathrm{CI}, 0.47-0.81, P=0.0005$; Figure 3C). There was evidence of publication bias by Egger's test $(P=0.029)$. However, single UFT chemotherapy did not show a DFS benefit with an HR of 1.04 (95\% CI, 0.76-1.43, $P=0.81$; Figure 3D) without publication bias. OS information was available for analysis in nine cisplatin-based trials (1,854 patients) and five single UFT trials (1,802 patients). Compared with 


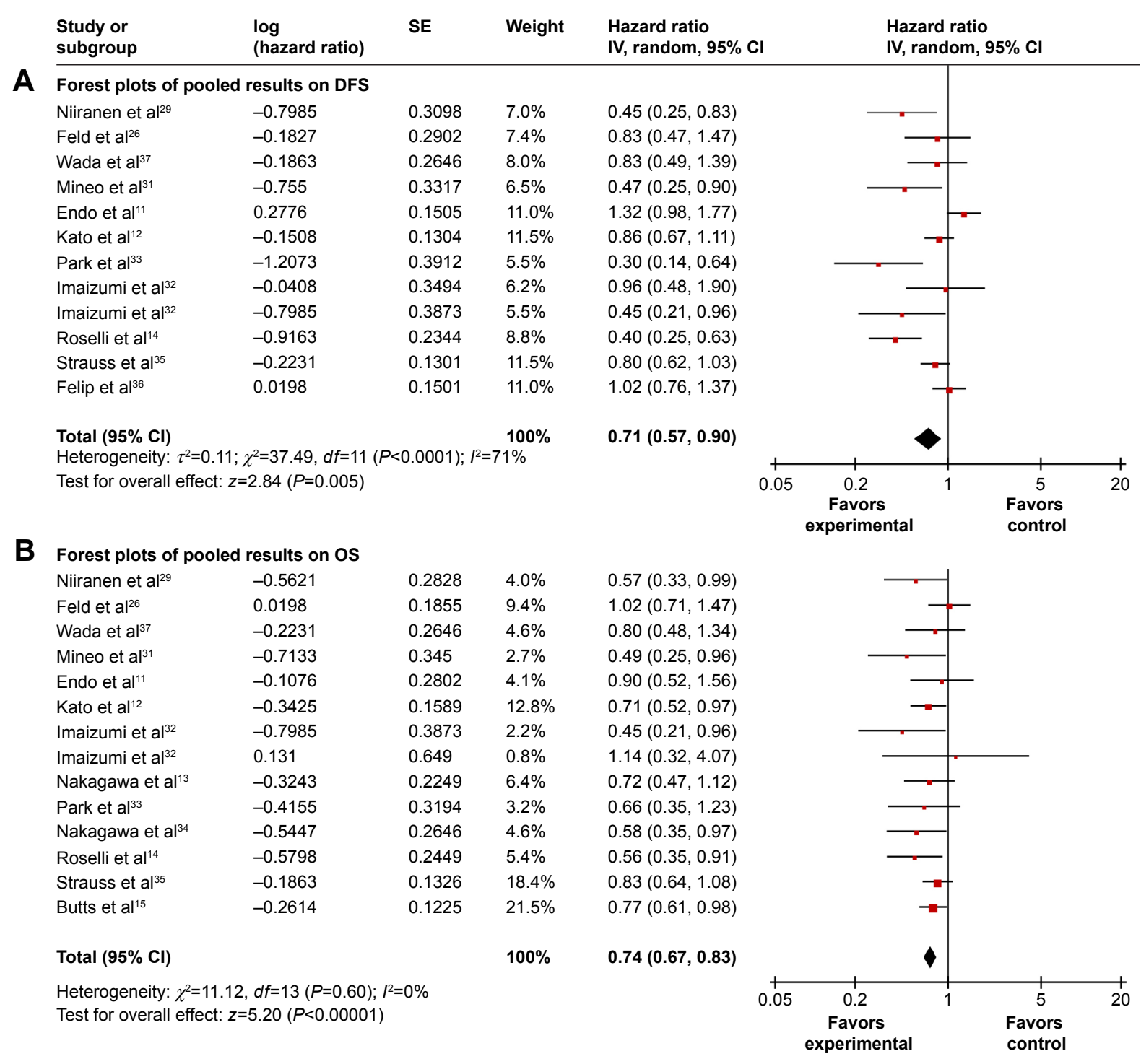

Figure 2 Forest plots of pooled results on DFS (A) and OS (B) comparing chemotherapy and control groups.

Abbreviations: $\mathrm{Cl}$, confidence interval; DFS, disease-free survival; IV, inverse variance; OS, overall survival; $\mathrm{SE}$, standard error.

control groups, both cisplatin-based (HR: $0.75, P<0.0001$; Figure 4D) and single UFT (HR: 0.72, $P=0.002$; Figure 4E) chemotherapy showed survival benefits in the combined OS analysis. Some evidence of publication bias was identified in DFS analysis of cisplatin-based chemotherapy by Begg's $(P=0.048)$ and Egger's tests $(P=0.045)$. ITC demonstrated that cisplatin-based chemotherapy had a longer DFS than single UFT chemotherapy (HR: $0.587,95 \%$ CI, $0.387-0.89, P=0.04$ ), but it failed to show difference of OS between the two regimen types (HR: 1.042, 95\% CI, 0.816-1.33, $P=0.90$ ).

\section{Toxicity analysis}

The toxicity was assessed in 1,692 (97\%) of 1,745 patients assigned to chemotherapy group. One trial did not collect toxicity data. ${ }^{15}$ Grade 3-4 neutropenia, nausea and vomiting, thrombocytopenia, and infection were observed in $16.4 \%$, $10.7 \%, 2 \%$, and $1.9 \%$ of the patients who received cisplatinbased chemotherapy, respectively. Incidence of other adverse effects like sensory neuropathy, anemia, and diarrhea were less than $1 \%$. Incidence of grade 3 or 4 nausea/vomiting and anorexia were observed in $0.8 \%$ and $0.7 \%$ of the patients who received single UFT chemotherapy, respectively. Only four treatment-related deaths $(0.2 \%)$ occurred in chemotherapy group (data not shown). These findings indicate that chemotherapy toxicity was mild and well tolerated.

\section{Sensitivity analysis and publication bias}

Although we applied a random effects model that took variation across studies into consideration, heterogeneity was still significant in DFS analysis. Therefore, we conducted 


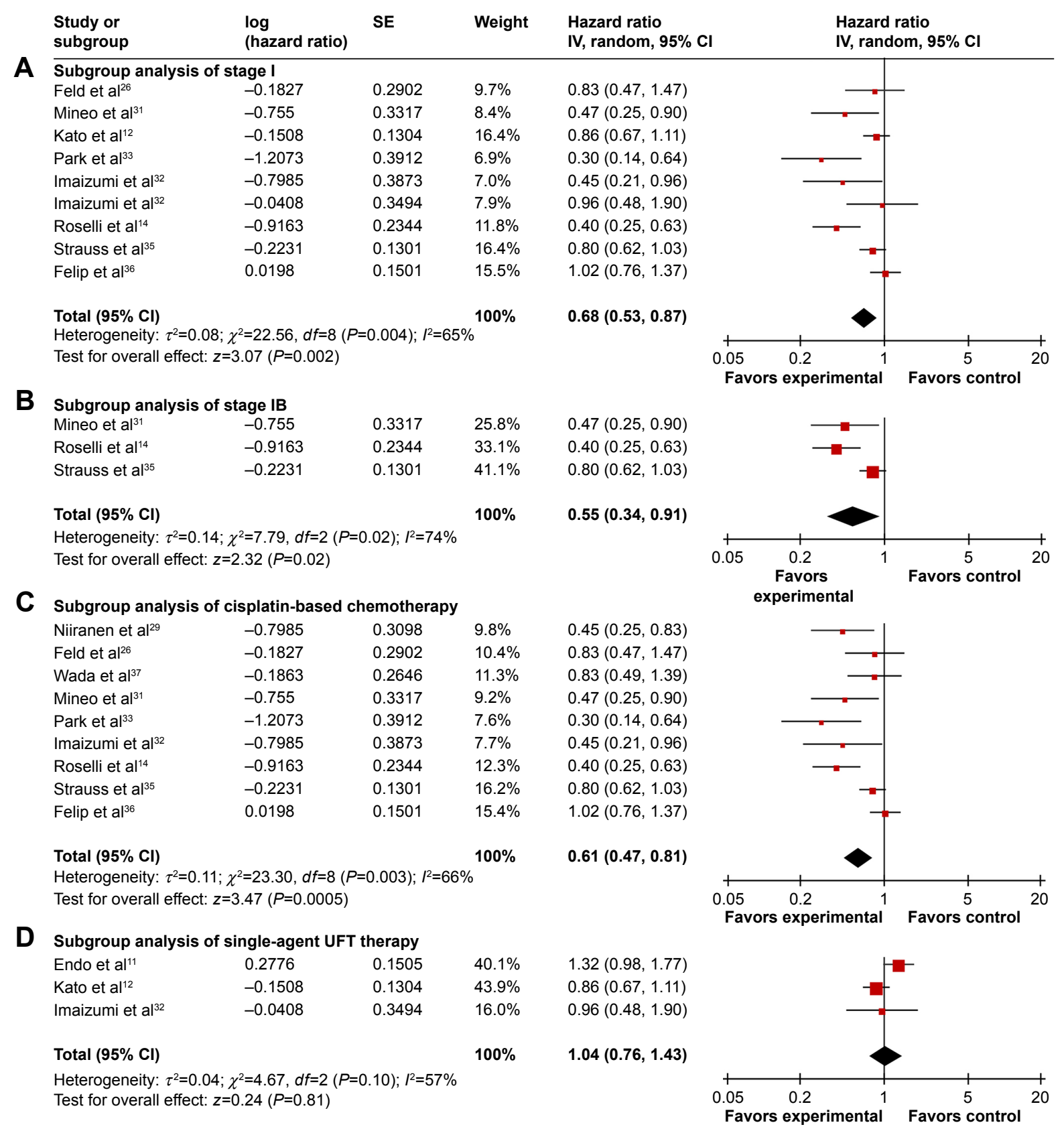

Figure 3 Subgroup analysis of DFS comparing chemotherapy and control groups.

Notes: (A) Subgroup analysis of stage I. (B) Subgroup analysis of stage IB. (C) Subgroup analysis of cisplatin-based chemotherapy. (D) Subgroup analysis of single-agent UFT therapy.

Abbreviations: $\mathrm{Cl}$, confidence interval; DFS, disease-free survival; IV, inverse variance; SE, standard error; UFT, tegafur-uracil.

sensitivity analysis to search for the source of heterogeneity. The findings showed some trials might influence integral heterogeneity, but the results of DFS did not essentially change after excluding these individual trials, so the results reported in this study were stable and acceptable. Publication bias by Begg's and Egger's tests is summarized in Table 2.

\section{Discussion}

As an important adjuvant treatment, the effect of postoperative chemotherapy in NSCLC is an important concern of oncologists. In recent years, several meta-analyses that explored the benefits of postoperative chemotherapy for NSCLC had been published, and the main characteristics are listed in Table 3. They demonstrated that postoperative chemotherapy was beneficial for NSCLC, but they did not discuss the survival benefit of chemotherapy without radiotherapy in early stage patients. Therefore, this meta-analysis was restricted to postoperative chemotherapy alone and early stage NSCLC.

We found survival advantages of receiving postoperative chemotherapy alone in early NSCLC patients. 


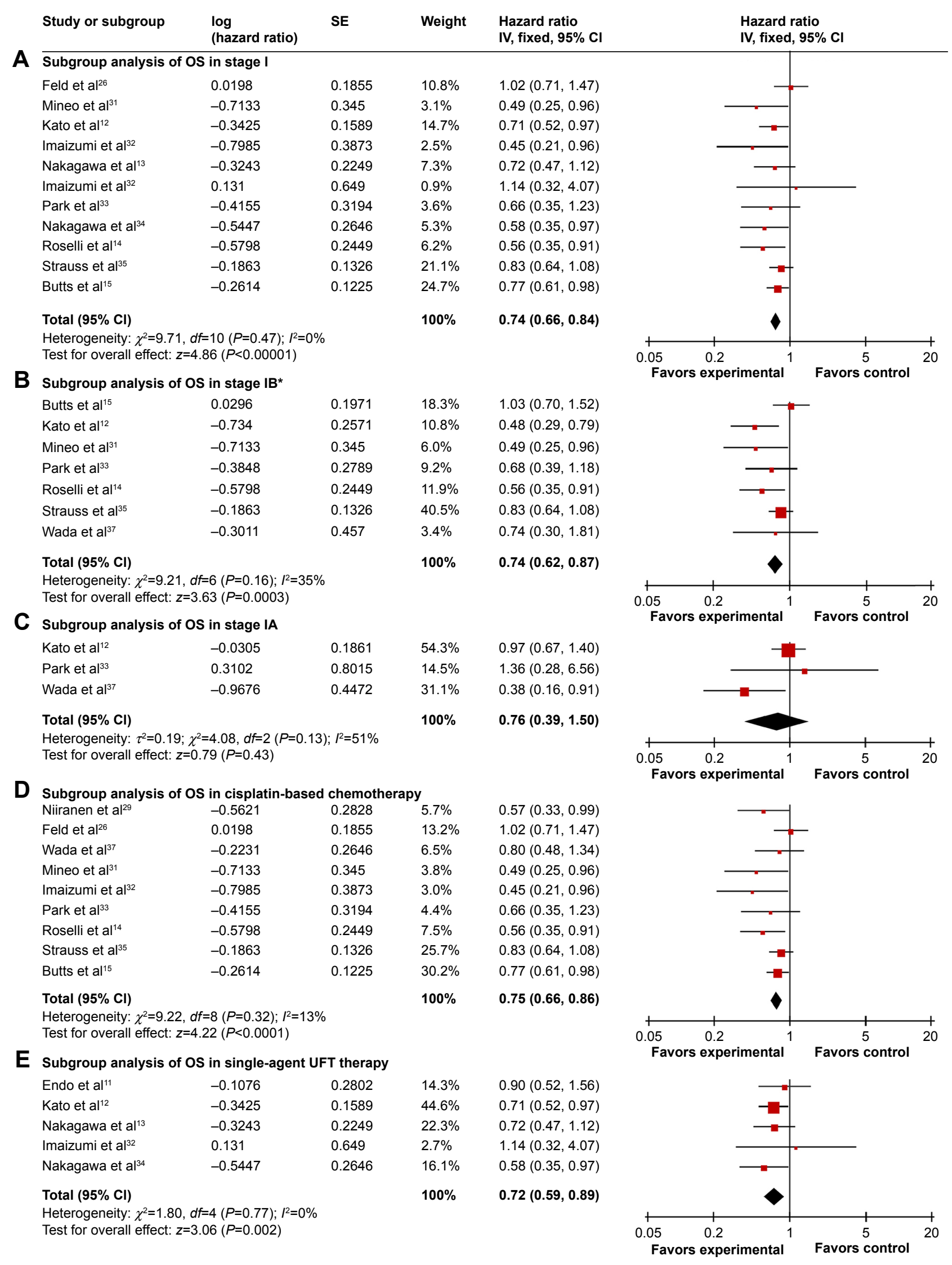

Figure 4 Subgroup analysis of OS comparing chemotherapy and control groups.

Notes: (A) Subgroup analysis of OS in stage I. (C) Subgroup analysis of OS in stage IA. (B) Subgroup analysis of OS in stage IB. (D) Subgroup analysis of OS in cisplatin-based chemotherapy. (E) Subgroup analysis of OS in a single-agent UFT therapy. *Used random effect models.

Abbreviations: $\mathrm{Cl}$, confidence interval; IV, inverse variance; OS, overall survival; SE, standard error; UFT, tegafur-uracil. 
Table 2 Publication bias accessed by Begg's and Egger's tests

\begin{tabular}{lll}
\hline & Begg's test & Egger's test \\
\hline DFS & & \\
Stage I-II & 0.304 & 0.029 \\
Stage I & $0.25 I$ & 0.065 \\
Stage IB & 1.000 & 0.336 \\
Cisplatin-based chemotherapy & 0.118 & 0.029 \\
Single UFT chemotherapy & 1.000 & 0.950 \\
OS & & \\
Stage I-II & 0.112 & 0.152 \\
Stage I & 0.087 & 0.152 \\
Stage IA & 1.000 & 0.771 \\
Stage IB & 0.548 & 0.187 \\
Cisplatin-based chemotherapy & 0.048 & 0.045 \\
Single UFT therapy & 0.462 & 0.402 \\
\hline
\end{tabular}

Abbreviations: DFS, disease-free survival; OS, overall survival; UFT, tegafururacil.
Subgroup analysis showed that this advantage had statistical significance in stages I and IB but not in stage IA. Similar results were also reported in other meta-analyses. Hamada et $\mathrm{al}^{38}$ focused on postoperative chemotherapy with UFT in stage I NSCLC and reported that 5-years OS rate was significantly higher in the chemotherapy group compared to control group ( $81.5 \%$ versus $77.2 \%, P=0.011$, HR: 0.74 ). Bria et $\mathrm{al}^{39}$ reported that patients with stage I-III disease who received cisplatin-based chemotherapy had a longer survival than those who underwent surgery alone in all stages including early NSCLC. However, the relative risk (instead of the more frequently used HR) and non-full paper trials in Bria's analysis might influence the accuracy of survival determination. Unlike stages IB and I, we did not observe

Table 3 Main results of other related meta-analyses

\begin{tabular}{|c|c|c|c|c|c|c|}
\hline \multirow[t]{2}{*}{ Meta-analyses } & \multirow[t]{2}{*}{ Objects } & \multirow[t]{2}{*}{ Radiotherapy } & \multicolumn{2}{|l|}{ Number } & \multirow[t]{2}{*}{ HR $(95 \% \mathrm{Cl})$} & \multirow[t]{2}{*}{$P$-value } \\
\hline & & & Studies & Patients & & \\
\hline \multirow[t]{4}{*}{ Hotta et al ${ }^{4}$} & Stage I-IV patients & Yes & 11 & 5,537 & $0.87(0.81-0.94)$ & 0.001 \\
\hline & Subgroup analyses & & & & & \\
\hline & $S$ versus $S+$ cis-based & Yes & 8 & 3,786 & $0.89(0.82-0.98)$ & 0.012 \\
\hline & $S$ versus $S+U F T$ & No & 5 & $|, 75|$ & $0.80(0.67-0.96)$ & 0.015 \\
\hline \multirow[t]{3}{*}{ Sedrakyan et al ${ }^{41}$} & Stage I-IIIA patients & Yes & 19 & 7,200 & $0.87(0.8 \mathrm{I}-0.93)$ & $<0.000$ I \\
\hline & $\mathrm{S}$ versus $\mathrm{S}+$ cis-based & Yes & 12 & NR & $0.89(0.82-0.96)$ & 0.004 \\
\hline & $S$ versus $S+U F T$ & No & 7 & NR & $0.87(0.81-0.93)$ & $<0.000$ I \\
\hline \multirow[t]{6}{*}{ Berghmans et al ${ }^{40}$} & Stage I-III patients & Yes & 19 & 7,644 & $0.84(0.78-0.89)$ & NR \\
\hline & Subgroup analyses ${ }^{\mathrm{a}}$ & & & & & \\
\hline & Stage I-II & Yes & 10 & 4,602 & $0.88(0.83-0.94)$ & NR \\
\hline & Stage III & Yes & 5 & $\mathrm{I}, 48 \mathrm{I}$ & $0.85(0.69-1.04)$ & NR \\
\hline & $\mathrm{S}$ versus $\mathrm{S}+$ cis-based & Yes & 16 & NR & $0.86(0.80-0.92)$ & NR \\
\hline & $S$ versus $S+U F T$ & No & 6 & NR & $0.72(0.61-0.85)$ & NR \\
\hline \multirow[t]{4}{*}{ Hamada et al ${ }^{38}$} & Stage I patients ${ }^{\mathrm{b}}$ & No & 6 & 2,003 & $0.74(0.61-0.88)$ & 0.001 \\
\hline & Subgroup analyses ${ }^{\mathrm{a}}$ & & & & & \\
\hline & $\mathrm{IA}$ & No & NR & I,308 & $0.73(0.56-0.93)$ & 0.72 \\
\hline & IB & No & NR & 674 & $0.78(0.60-1.01)$ & 0.72 \\
\hline \multirow[t]{6}{*}{ Pignon et al ${ }^{10}$} & Stage I-III patients ${ }^{c}$ & Yes & 5 & 4,584 & $0.89(0.82-0.96)$ & 0.005 \\
\hline & Subgroup analyses & & & & & \\
\hline & Stage IA & Yes & NR & NR & $1.40(0.95-2.06)$ & NR \\
\hline & Stage IB & Yes & NR & NR & $0.93(0.78-1.10)$ & NR \\
\hline & Stage II & Yes & NR & NR & $0.83(0.73-0.95)$ & NR \\
\hline & Stage III & Yes & NR & NR & $0.83(0.72-0.94)$ & NR \\
\hline \multirow[t]{5}{*}{ Bria et al ${ }^{39}$} & Stage I-III patients ${ }^{c}$ & Yes & 20 & 7,408 & $0.91(0.85-0.97)$ & 0.011 \\
\hline & Subgroup analyses ${ }^{\mathrm{a}}$ & & & & & \\
\hline & Stage I-II & Yes & 9 & 3,774 & $0.85(0.79-0.91)$ & $<0.000$ I \\
\hline & Stage I & Yes & 7 & I,888 & $0.88(0.79-0.98)$ & 0.022 \\
\hline & Stage III & Yes & 6 & 1,666 & $0.91(0.85-0.96)$ & 0.004 \\
\hline NSCLC & Stage I-IV patients & Yes & 34 & 8,447 & $0.86(0.81-0.92)$ & $<0.000$ I \\
\hline Meta-analyses & Subgroup analyses ${ }^{\mathrm{a}}$ & & & & & \\
\hline Collaborative & $S$ versus $S+$ cis-based & Yes & 18 & 2,620 & $0.89(0.82-0.97)$ & 0.006 \\
\hline Group ${ }^{19}$ & $S$ versus $S+U F T$ & No & 8 & 3,848 & $0.80(0.71-0.90)$ & 0.001 \\
\hline
\end{tabular}

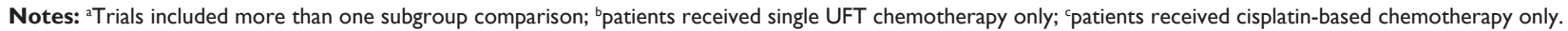
Abbreviations: HR, hazard ratio; OS, overall survival; Cl, confidence interval; S, surgery; UFT, tegafur-uracil; NR, not reported; cis-based, cisplatin-based chemotherapy. 
an OS benefit of chemotherapy for stage IA disease (HR: $0.76,95 \% \mathrm{CI}, 0.39-1.50)$. This result was compatible with the Lung Adjuvant Cisplatin Evaluation Collaborative Group, ${ }^{10}$ which performed a trial on cisplatin-based chemotherapy in patients with stage I-III disease. Negative efficacy of postoperative chemotherapy in stage IA was based on only three trials, likely because stage IA patients are rare at initial surgery and these patients are less likely to receive postoperative chemotherapy. These results need to be confirmed in large RCTs.

Consistent with the study conducted by Berghmans et a ${ }^{40}$ we found that both cisplatin-based and single UFT chemotherapy significantly improved OS. But their analysis included two trials presented at the American Society of Clinical Oncology (ASCO) Annual Meeting, which might cause the imprecise effect because of insufficient data (ie, randomization procedures, patient allocation, or trials quality). A meta-analysis including stage I-III patients conducted by Hotta et $\mathrm{al}^{4}$ also suggested that both cisplatin-based chemotherapy (HR: $0.89, P=0.012$ ) and single UFT (HR: 0.80, $P=0.015$ ) significantly improved OS. This postoperative chemotherapy benefit in OS was also confirmed by Sedrakyan et al. ${ }^{41}$ These data strengthened our results regarding subgroup analysis of different chemotherapy regimens. Importantly, advantages of cisplatin-based and single UFT chemotherapy identified in our study were superior to those reported in other meta-analyses. It should be noted that the above three meta-analyses did not investigate the effect of postoperative chemotherapy on DFS, while we did it. Our results demonstrated that cisplatin-based chemotherapy significantly improved DFS, but single UFT chemotherapy did not. In addition, we provided the first evidence that cisplatin-based chemotherapy was better than single UFT chemotherapy with regard to DFS for early NSCLC patients by ITC software, but the OS benefits were similar for the two kinds of chemotherapy regimens. However, this finding should be interpreted carefully, because all the UFT trials were conducted in Japan and this agent was not extensively administrated in other areas. In addition, the small number of studies was not sufficient to draw exact conclusions. More RCTs about postoperative UFT chemotherapy for NSCLC outside Japan should be conducted in the future.

The PORT meta-analysis ${ }^{16}$ indicated that postoperative radiotherapy had a detrimental effect on survival, particularly for early stage NSCLC patients. So, it was unreasonable that postoperative radiotherapy was included in the meta-analysis of postoperative chemotherapy, because it might increase chemotherapy toxicities. However, these meta-analyses listed in Table 3 still permitted postoperative radiotherapy in selecting eligible trials, including the study conducted by NSCLC Meta-analyses Collaborative Group. ${ }^{19}$ For the reasons mentioned above, trials with postoperative radiotherapy were excluded from our meta-analysis to avoid accumulated harmful effects.

It should be noted that publication bias is a potential threat to the validity of our meta-analysis. Heterogeneity decreased after excluding studies by Endo et $\mathrm{al}^{11}$ and Roselli et a ${ }^{14}$ from the overall DFS analysis; Roselli et a $1^{14}$ and Felip et al ${ }^{36}$ from stage I analysis; and Strauss et $\mathrm{al}^{35}$ and Felip et $\mathrm{al}^{36}$ from cisplatin-based chemotherapy analysis. The 5-year OS rate of control group was $75 \%$ in the study by Endo et a ${ }^{11}$ which was higher than the expected survival rate $(50 \%)$ due to the majority of IA patients. So, the number of eligible cases $(n=122)$ in trial design was too small to detect a difference. The study by Felip et $\mathrm{al}^{36}$ was conducted in several centers and the compliance was only $51 \%$. The study by Strass et $\mathrm{al}^{35}$ stopped before pre-assignment time. In addition, a subset of the patients underwent minimal resection but not complete resection in the study by Roselli et al. ${ }^{14}$ Additionally, these trials that had large age difference with others were excluded from overall heterogeneity analysis. The value of $I^{2}$ was still $>50 \%$ after excluding studies with minimum ${ }^{29}$ and maximum age, ${ }^{14}$ and two studies with age $<60$ years. ${ }^{29,33}$ Therefore, age did not substantially influence heterogeneity of DFS. These factors may explain the different results among the studies.

Several limitations in this meta-analysis should be pointed out. Firstly, heterogeneity existed in some of the DFS results. Some factors, such as variable ethnic origin, TNM stage, age, and drug administration, could explain some of the difference among the studies. Secondly, we confirmed the superiority of postoperative chemotherapy alone in early stage NSCLC patients, but only $51 \%-86 \%$ of patients completed their predesigned chemotherapy regimens and there was an obvious gap of compliance among all trials. Further efforts to improve compliance without increasing toxicities are essential. Lastly, only articles published in full papers and English language were included in this meta-analysis. This selection might induce a potential publication bias, because positive studies are more likely and easier to be published than negative ones.

\section{Conclusion}

In conclusion, our meta-analysis demonstrates the positive efficacy of postoperative chemotherapy alone in stage I-II, I, and IB NSCLC with mild toxicity, but a significant benefit was not found in IA patients. Meanwhile, this meta-analysis 
also indicates that efficacy of cisplatin-based chemotherapy is comparable to single UFT chemotherapy in OS, but better than single UFT chemotherapy in DFS; however, further studies are needed to verify these findings in clinical practice. In view of few trials that have assessed the effects of postoperative chemotherapy alone in stage IA and II patients, we suggest that more trials should be conducted to confirm the effectivity of postoperative chemotherapy in stage IA and II NSCLC patients in future.

\section{Acknowledgment}

This work was supported by National High Technology Research and Development Program of China [grant number: 2012AA02A502]. The funders had no role in study design, data collection and analysis, decision to publish, or preparation of the manuscript.

\section{Disclosure}

The authors declared no conflict of interest.

\section{References}

1. Cheng X, Chen H. Tumor heterogeneity and resistance to EGFRtargeted therapy in advanced nonsmall cell lung cancer: challenges and perspectives. Onco Targets Ther. 2014;7:1689-1704.

2. Jemal A, Bray F, Center MM, Ferlay J, Ward E, Forman D. Global cancer statistics. CA Cancer J Clin. 2011;61(2):69-90.

3. Molina JR, Yang P, Cassivi SD, Schild SE, Adjei AA. Non-small cell lung cancer: epidemiology, risk factors, treatment, and survivorship. Mayo Clin Proc. 2008;83(5):584-594.

4. Hotta K, Matsuo K, Ueoka H, Kiura K, Tabata M, Tanimoto M. Role of adjuvant chemotherapy in patients with resected non-small-cell lung cancer: reappraisal with a meta-analysis of randomized controlled trials. J Clin Oncol. 2004;22(19):3860-3867.

5. Thomas P, Doddoli C, Thirion X, et al. Stage I non-small cell lung cancer: a pragmatic approach to prognosis after complete resection. Ann Thorac Surg. 2002;73(4):1065-1070.

6. Karim-Kos HE, de Vries E, Soerjomataram I, Lemmens V, Siesling S, Coebergh JW. Recent trends of cancer in Europe: a combined approach of incidence, survival and mortality for 17 cancer sites since the 1990 s. Eur J Cancer. 2008;44(10):1345-1389.

7. Chansky K, Sculier JP, Crowley JJ, et al. The International Association for the Study of Lung Cancer Staging Project: prognostic factors and pathologic TNM stage in surgically managed non-small cell lung cancer. J Thorac Oncol. 2009;4(7):792-801.

8. Pfister DG, Johnson DH, Azzoli CG, et al. American Society of Clinical Oncology treatment of unresectable non-small-cell lung cancer guideline: update 2003. J Clin Oncol. 2004;22(2):330-353.

9. Baggstrom MQ, Stinchcombe TE, Fried DB, Poole C, Hensing TA, Socinski MA. Third-generation chemotherapy agents in the treatment of advanced non-small cell lung cancer: a meta-analysis. $J$ Thorac Oncol. 2007;2(9):845-853.

10. Pignon JP, Tribodet H, Scagliotti GV, et al. Lung adjuvant cisplatin evaluation: a pooled analysis by the LACE Collaborative Group. J Clin Oncol. 2008;26(21):3552-3559.

11. Endo C, Saito Y, Iwanami H, et al. A randomized trial of postoperative UFT therapy in p stage I, II non-small cell lung cancer: North-east Japan Study Group for Lung Cancer Surgery. Lung Cancer. 2003;40(2): 181-186.
12. Kato $\mathrm{H}$, Ichinose $\mathrm{Y}$, Ohta $\mathrm{M}$, et al. A randomized trial of adjuvant chemotherapy with uracil-tegafur for adenocarcinoma of the lung. N Engl J Med. 2004;350(17):1713-1721.

13. Nakagawa M, Tanaka F, Tsubota N, et al. A randomized phase III trial of adjuvant chemotherapy with UFT for completely resected pathological stage I non-small-cell lung cancer: the West Japan Study Group for Lung Cancer Surgery (WJSG) - the 4th study. Ann Oncol. 2005; 16(1):75-80.

14. Roselli M, Mariotti S, Ferroni P, et al. Postsurgical chemotherapy in stage IB nonsmall cell lung cancer: Long-term survival in a randomized study. Int J Cancer. 2006;119(4):955-960.

15. Butts CA, Ding K, Seymour L, et al. Randomized phase III trial of vinorelbine plus cisplatin compared with observation in completely resected stage IB and II non-small-cell lung cancer: updated survival analysis of JBR-10. J Clin Oncol. 2010;28(1):29-34.

16. No authors listed. Postoperative radiotherapy in non-small-cell lung cancer: systematic review and meta-analysis of individual patient data from nine randomised controlled trials. PORT Meta-analysis Trialists Group. Lancet. 1998;352(9124):257-263.

17. Scagliotti GV, Fossati R, Torri V, et al. Randomized study of adjuvant chemotherapy for completely resected stage I, II, or IIIA non-small-cell Lung cancer. J Natl Cancer Inst. 2003;95(19):1453-1461.

18. Arriagada R, Bergman B, Dunant A, et al. Cisplatin-based adjuvant chemotherapy in patients with completely resected non-small-cell lung cancer. N Engl J Med. 2004;350(4):351-360.

19. NSCLC Meta-analyses Collaborative Group; Arriagada R, Auperin A, et al. Adjuvant chemotherapy, with or without postoperative radiotherapy, in operable non-small-cell lung cancer: two meta-analyses of individual patient data. Lancet. 2010;375(9722):1267-1277.

20. No authors listed. Chemotherapy in non-small cell lung cancer: a metaanalysis using updated data on individual patients from 52 randomised clinical trials. Non-small Cell Lung Cancer Collaborative Group. BMJ. 1995;311(7010):899-909.

21. Tierney JF, Stewart LA, Ghersi D, Burdett S, Sydes MR. Practical methods for incorporating summary time-to-event data into meta-analysis. Trials. 2007;8:16.

22. Higgins JP, Altman DG, Gøtzsche PC, et al. The Cochrane Collaboration's tool for assessing risk of bias in randomised trials. BMJ. 2011; 343:d5928.

23. Bucher HC, Guyatt GH, Griffith LE, Walter SD. The results of direct and indirect treatment comparisons in meta-analysis of randomized controlled trials. J Clin Epidemiol. 1997;50(6):683-691.

24. Begg CB, Mazumdar M. Operating characteristics of a rank correlation test for publication bias. Biometrics. 1994;50(4):1088-1101.

25. Egger M, Davey Smith G, Schneider M, Minder C. Bias in meta-analysis detected by a simple, graphical test. BMJ. 1997;315(7109):629-634.

26. Feld R, Rubinstein L, Thomas PA. Adjuvant chemotherapy with cyclophosphamide, doxorubicin, and cisplatin in patients with completely resected stage I non-small-cell lung cancer. The Lung Cancer Study Group. J Natl Cancer Inst. 1993;85(4):299-306.

27. Winton T, Livingston R, Johnson D, et al. Vinorelbine plus cisplatin vs. observation in resected non-small-cell lung cancer. $N$ Engl J Med. 2005; 352(25):2589-2597.

28. Feld R, Rubinstein L, Thomas PA. Adjuvant chemotherapy with cyclophosphamide, doxorubicin, and cisplatin in patients with completely resected stage I non-small cell lung cancer. An LCSG Trial. Chest. 1994;106(6 Suppl):307S-309S.

29. Niiranen A, Niitamo-Korhonen S, Kouri M, Assendelft A, Mattson K, Pyrhönen S. Adjuvant chemotherapy after radical surgery for non-small-cell lung cancer: a randomized study. J Clin Oncol. 1992;10(12):1927-1932.

30. Wada H, Hitomi S, Teramatsu T. Adjuvant chemotherapy after complete resection in non-small-cell lung cancer. West Japan Study Group for Lung Cancer Surgery. J Clin Oncol. 1996;14(4):1048-1054.

31. Mineo TC, Ambrogi V, Corsaro V, Roselli M. Postoperative adjuvant therapy for stage IB non-small-cell lung cancer. Eur J Cardiothorac Surg. 2001;20(2):378-384. 
32. Imaizumi M; Study Group of Adjuvant Chemotherapy for Lung Cancer (Chubu, Japan). Postoperative adjuvant cisplatin, vindesine, plus uracil-tegafur chemotherapy increased survival of patients with completely resected p-stage I non-small cell lung cancer. Lung Cancer. 2005;49(1):85-94

33. Park JH, Lee CT, Lee HW, Baek HJ, Zo JI, Shim YM. Postoperative adjuvant chemotherapy for stage I non-small cell lung cancer. Eur J Cardiothorac Surg. 2005;27(6):1086-1091.

34. Nakagawa K, Tada H, Akashi A, et al. Randomised study of adjuvant chemotherapy for completely resected p-stage I-IIIA non-small cell lung cancer. Br J Cancer. 2006;95(7):817-821.

35. Strauss GM, Herndon JE 2nd, Maddaus MA, et al. Adjuvant paclitaxel plus carboplatin compared with observation in stage IB non-small-cell lung cancer: CALGB 9633 with the Cancer and Leukemia Group B, Radiation Therapy Oncology Group, and North Central Cancer Treatment Group Study Groups. J Clin Oncol. 2008;26(31):5043-5051.

36. Felip E, Rosell R, Maestre JA, et al. Preoperative chemotherapy plus surgery versus surgery plus adjuvant chemotherapy versus surgery alone in early-stage non-small-cell lung cancer. J Clin Oncol. 2010;28(19): 3138-3145.
37. Wada H, Miyahara R, Tanaka F, Hitomi S. Postoperative adjuvant chemotherapy with PVM (Cisplatin + Vindesine + Mitomycin C) and UFT (Uracil + Tegaful) in resected stage I-II NSCLC (non-small cell lung cancer): a randomized clinical trial. West Japan Study Group for lung cancer surgery (WJSG). Eur J Cardiothorac Surg. 1999;15(4): $438-443$.

38. Hamada C, Tanaka F, Ohta M, et al. Meta-analysis of postoperative adjuvant chemotherapy with tegafur-uracil in non-small-cell lung cancer. J Clin Oncol. 2005;23(22):4999-5006.

39. Bria E, Gralla RJ, Raftopoulos H, et al. Magnitude of benefit of adjuvant chemotherapy for non-small cell lung cancer: meta-analysis of randomized clinical trials. Lung Cancer. 2009;63(1):50-57.

40. Berghmans T, Paesmans M, Meert AP, et al. Survival improvement in resectable non-small cell lung cancer with (neo)adjuvant chemotherapy: results of a meta-analysis of the literature. Lung Cancer. 2005; 49(1):13-23

41. Sedrakyan A, Van Der Meulen J, O’Byrne K, Prendiville J, Hill J, Treasure T. Postoperative chemotherapy for non-small cell lung cancer: a systematic review and meta-analysis. J Thorac Cardiovasc Surg. 2004; $128(3): 414-419$.
OncoTargets and Therapy

\section{Publish your work in this journal}

OncoTargets and Therapy is an international, peer-reviewed, open access journal focusing on the pathological basis of all cancers, potential targets for therapy and treatment protocols employed to improve the management of cancer patients. The journal also focuses on the impact of management programs and new therapeutic agents and protocols on

\section{Dovepress}

patient perspectives such as quality of life, adherence and satisfaction. The manuscript management system is completely online and includes a very quick and fair peer-review system, which is all easy to use. Visit http://www.dovepress.com/testimonials.php to read real quotes from published authors. 